\title{
Proposal of a Device Detecting Two Dimensional Coordinates Using PSD Sensor and Ultrasonic Sensor
}

\author{
Yuya Kawahara $^{\mathrm{a}, *}$, Ryuichi Udo ${ }^{\mathrm{a}}$, and Shiyuan Yang ${ }^{\mathrm{a}}$ \\ ${ }^{a}$ Kyushu Institute of Technology, 1-1 Sensui-cho Tobata-ku Kitakyushu-shi Fukuoka-ken, 804-8550, \\ Japan
}

*Corresponding Author. kawahara@ boss.ecs.kyutech.ac.jp

\begin{abstract}
Currently, the touch panel is used in various products. However, the size of the device is determined by the size of the touch panel. As a touch panel which is not to depend on the device size, the projection type touch panel exists. However, the projector is difficult to set to anywhere. Recently, devices called laser keyboard have been proposed. It can be used as a touch panel using the method of any space by using the laser and the optical sensor. However, miniaturization is difficult because it uses the principle of triangulation to detect the finger position. In this study, we propose a two-dimensional coordinate acquiring device using ultrasonic sensors and PSD sensor there. Without using triangulation by combining the optical sensor and the ultrasonic sensor, two-dimensional coordinates of the finger against any surface can be acquired. Since our device does not need a distance between the sensor and the laser, the size of the product employing our device can be shrunken more. In other words, our device can be embedded to the small factor product easily.
\end{abstract}

Keywords: PSD, ultrasonic sensor, touch panel.

\section{Introduction}

Now, the touch panel is used in various electronic devices, such as a smart phone, ATM, and so on. Since touch panels display the key on the display, they can arbitrarily set the size of the button and the shape within the display range. However, by this technique, the area which can be used as a touch panel is dependent on the size of equipment. Thus, to avoid the operation mistake when placing many buttons in the viewable area, the equipment cannot become small.
As a touch panel which is not to depend on the device size, the projection type touch panel exists ${ }^{[1]}$. However, the projector is difficult to set to anywhere.

A laser keyboard is mentioned as a method of solving this problem ${ }^{[2]}$. By using the laser and sensor sensing the reflected laser from the fingers, the laser keyboard measures the two-dimensional coordinates of the position of the finger by triangulation. Another laser projects the button onto the surface as a touch panel. Therefore, create a virtual two-dimensional touch panel in any plane. In this technique, the usable range does not depend on the size of the device as a touch panel. Thus, the device itself is small but the touch panel used can be a wide range. However, since the measurement is performed using a triangulation, it is necessary to a certain distance between the laser and the sensor. That is, this device is a limit to further miniaturization.

To realize the touch panel which does not depend on the device size for the viewable area and can make the product size smaller, we propose a two-dimensional coordinate acquisition device using a PSD sensor and ultrasonic sensors. This device obtains the angle which is constructed by the optical axis of the lens and the straight-line on the finger to the sensor ${ }^{[3]}$. An ultrasonic sensor measures the distance between a finger and the device. By combining the angle and distance measured, the two-dimensional coordinates of the finger on the surface can be determined. Since our proposal does not use the triangulation to determine the finger position, the distance between the ultrasonic sensor and a light receiving sensor in the same device can be significant short. That is, principally, our proposal can measure the finger position on the one point with zero size of area.

Below, we describe our proposal briefly and then report 
the experimental results measuring two-dimensional coordinates of the finger practically.

\section{Proposed System}

\subsection{System Organization}

The system configuration of this research is shown in Fig. 1. First, the device is placed on a flat surface you want to get the two-dimensional coordinates. An ultrasonic wave is generated from an ultrasonic transmission element. The reflected wave from the finger is received with an ultrasonic receiving element. The distance $r$ from the equipment to a finger is calculated by the microcomputer using ultrasonic propagation time, and a calculated result is transmitted to a personal computer by serial communication. Furthermore, as shown in Fig. 1, the laser light is irradiated to a rod lens at right angles to the axis of a lens. Through the rod lens, the light extends in a fan fashion above $10 \mathrm{~mm}$ from the field used as the touch panel. When the finger blocks the light, the laser light is irradiated to the finger. The reflected light through the lens reaches the PSD. By determining the coordinates of the center of gravity of the light irradiated to the PSD, the angle $\theta$ can be calculated by the optical axis of the lens and the line connecting the fingers and PSD shown in Figure 1.

The two-dimensional coordinates of the position of the finger from the distance $r$ and angle $\theta$ obtained by these operations can be identified.

\subsection{Ultrasonic Transmitter Circuit}

To detect the distance between the device and the user's finger, an ultrasonic sensor is used. This section describes the detail of the ultrasonic wave transmitter. Henceforth, we assume the supply voltage $(V c c)$ is $5 \mathrm{~V}$.

(a) Pulse wave oscillation circuit

A pulse wave oscillation circuit using a timer IC shown in Figure 2 synchronizes the ultrasonic receiving circuit. In this case, $R_{l}=3.3[\mathrm{M} \Omega], R_{2}=47[\mathrm{k} \Omega], C_{l}=0.01[\mu \mathrm{F}]$, and $C_{2}=0.1[\mu \mathrm{F}]$. Figure 3 shows the pulse waveform generated by this circuit. The $T_{L}$ is the time during the pulse wave is low. The $T_{H}$ is the time during the pulse wave is high. $T_{L}$ and $T_{H}$ can be determined by the following equations (1) and (2).

$$
\begin{aligned}
& T_{L}=0.69 \times\left(R_{1}+R_{2}\right) \times C_{1} \\
& T_{H}=0.69 \times R_{2} \times C_{1}
\end{aligned}
$$

By inserting values into the variables of equation(1)

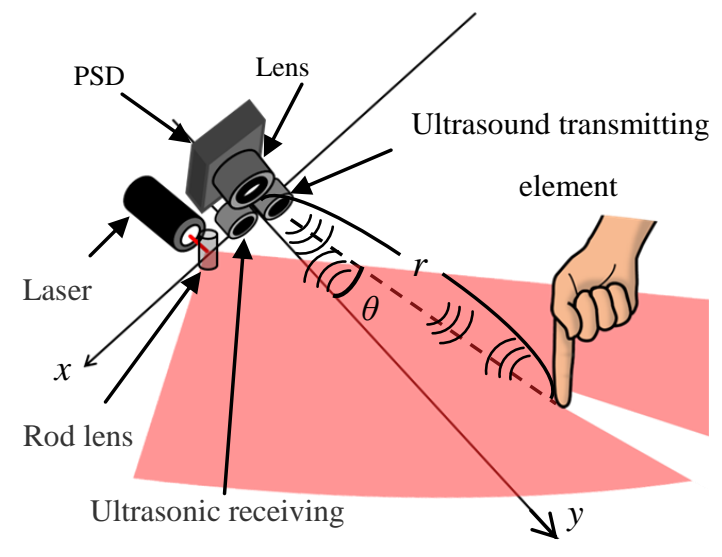

element

Fig. 1. System Organization.

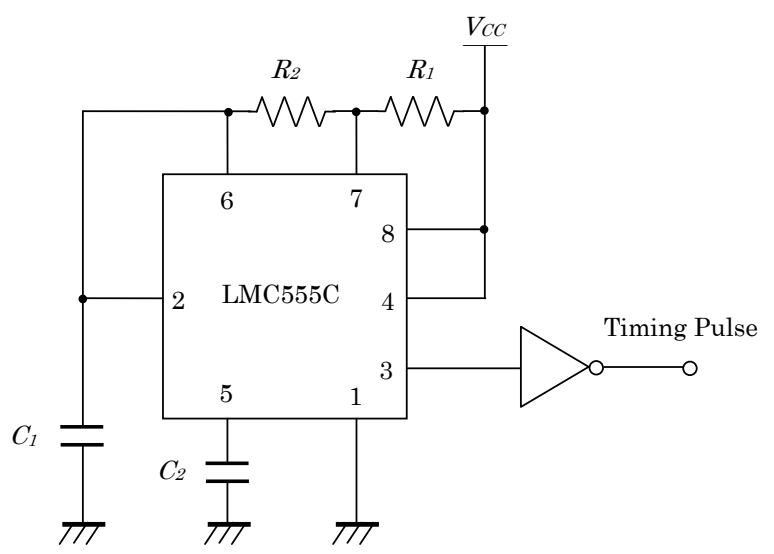

Fig. 2. Pulse wave oscillation circuit.

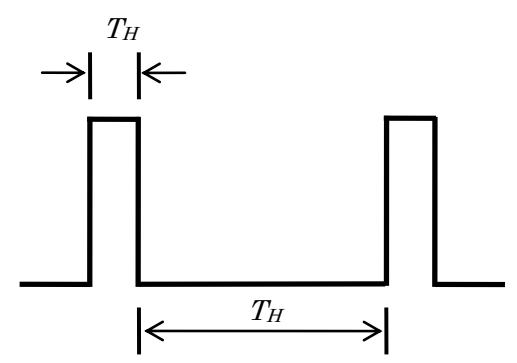

Fig. 3. Meter pulse generated by the pulse oscillator circuit.

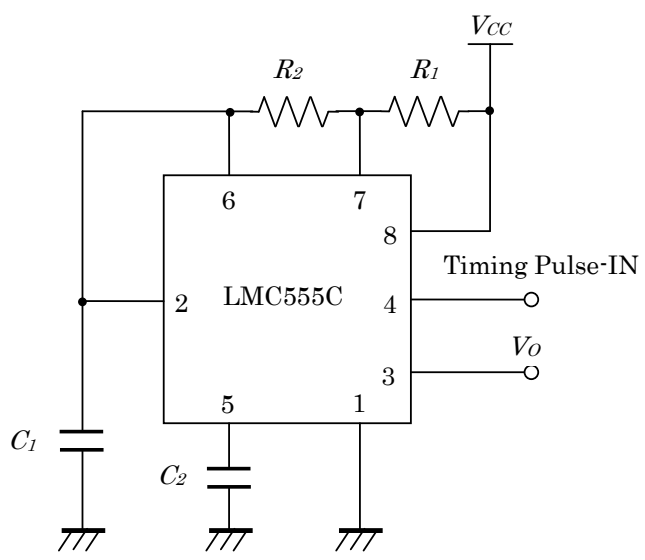

Fig. 4. Frequency modulation circuit. 
and (2), we get TL and TH as follows.

$$
\begin{gathered}
T_{L}=0.69 \times\left(3.3 \times 10^{6}+47 \times 10^{3}\right) \times 0.01 \\
\times 10^{-6}=23.09[\mathrm{~ms}] \\
T_{H}=0.69 \times 47 \times 10^{3} \times 0.01 \times 10^{-6} \\
=0.3243[\mathrm{~ms}]
\end{gathered}
$$

Pulse period is $23 \mathrm{~ms}$ and the pulse width is $0.3 \mathrm{~ms}$.

\section{(b) Frequency modulation circuit}

We design a frequency modulation circuit using a timer IC, to modulate the frequency of the ultrasound. This circuit is shown in Figure 4. In this case, $R_{1}=1.5[\mathrm{k} \Omega], R_{2}=15$ $[\mathrm{k} \Omega], C_{1}=1000[\mathrm{pF}]$, and $C_{2}=0.1[\mu \mathrm{F}]$. Unlike Figure 2, by giving the pin 4 of the timer IC a timing pulse instead of the power supply voltage $V_{C C}$, the pulse wave frequency modulation is performed only when the timing pulse is high.

This makes it possible to send ultrasonic waves between $0.3 \mathrm{~ms}$ every $25 \mathrm{~ms}$ of $T_{L}$ and $T_{H}$ shown in Figure.3. Oscillation frequency can be obtained by the equation (5).

$$
f=\frac{1}{T_{L}+T_{H}}
$$

$T_{H}, T_{L}$ and $\mathrm{f}$ are calculated by assigning a value to each of the formula (5), (1) and (2) .

$$
\begin{gathered}
T_{L}=0.69 \times\left(1.5 \times 10^{3}+15 \times 10^{3}\right) \times 1000 \\
\times 10^{-12}=11.385[\mu \mathrm{s}] \\
T_{H}=0.69 \times 15 \times 10^{3} \times 1000 \times 10^{-12} \\
=10.35[\mu \mathrm{s}] \\
f=\frac{1}{11.385 \times 10^{-6}+10.35 \times 10^{-6}} \\
=46[\mathrm{kHz}]
\end{gathered}
$$

The oscillation frequency is $46 \mathrm{kHz}$ from equation (8). Thus, we can generate a frequency which is sufficient to generate an ultrasonic wave.

(c) Ultrasonic oscillation circuit

To strengthen the transmission output, we design an ultrasonic oscillator circuit using NOT circuit as shown in Figure 5. In this case, $C=0.1[\mu \mathrm{F}]$.

Figure 6 shows the output voltage across the terminals at both ends of the ultrasonic sensor - + terminal. Since the outputs of the positive and negative terminals are summed up, the voltage applied to both ends of the ultrasonic sensor is twice of the input voltage. Thus, we can increase the output strength from the ultrasonic sensor ultrasonic wave transmission.

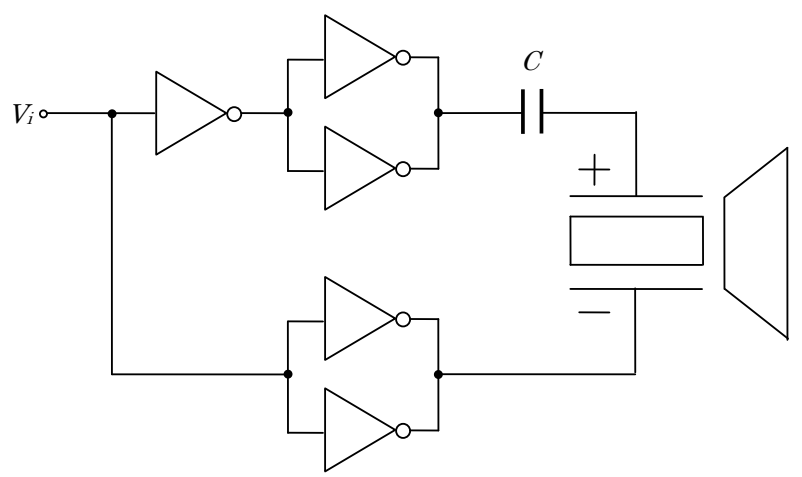

Fig. 5. Ultrasonic oscillation circuit.
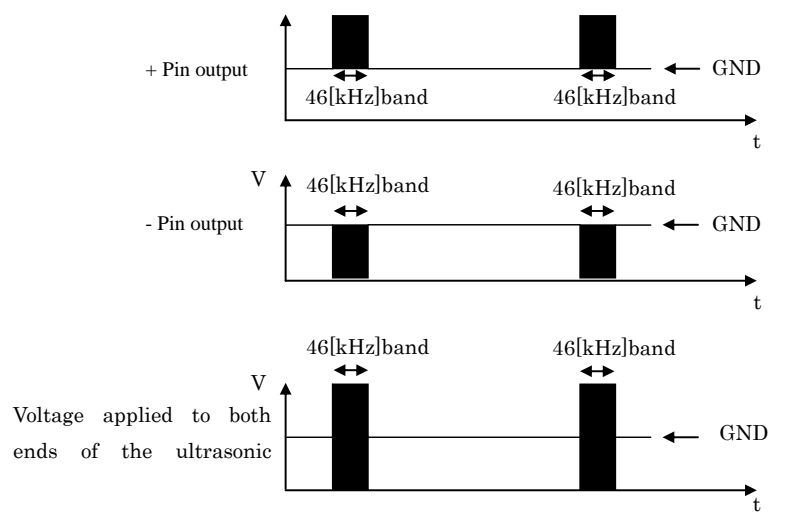

Fig. 6. Total output of the oscillation circuit ultrasonic wave.

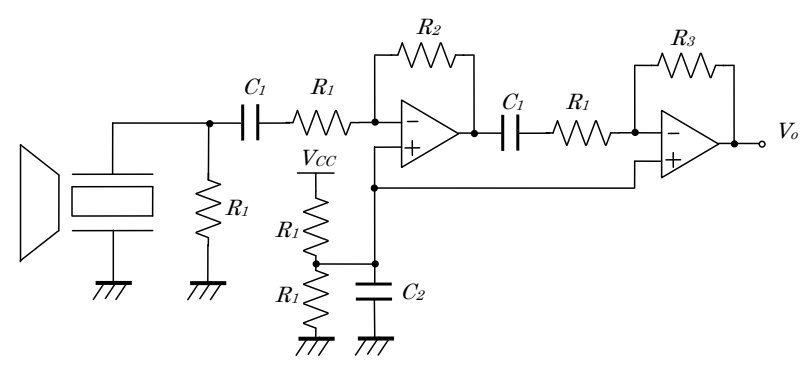

Fig. 7. Signal amplification circuit.

\subsection{Ultrasonic Receiver Circuit}

This section describes the receiving circuit of the ultrasonic wave transmitted from the ultrasonic transmitter circuit.

(a) Signal amplification circuit

The ultrasonic receiver amplifies the signal as shown in Figure 7. In this case, $R_{l}=10[\mathrm{k} \Omega], R_{2}=1[\mathrm{M} \Omega], R_{3}=100$ $[\mathrm{k} \Omega], C_{l}=1000[\mathrm{pF}]$, and $C_{2}=0.1[\mu \mathrm{F}]$.

This amplifier is the two-stage operational amplifier. The amplification factor is 10 times at the first-stage (20 $[\mathrm{dB}])$ and the second stage is 100 times $(40[\mathrm{~dB}])$. Totally, it amplifies the input signal by 1000 times $(60[\mathrm{~dB}])$.

In addition, the reference voltage of this amp is $2.5 \mathrm{~V}$. 


\section{(b) Detector circuit}

We design a half-wave rectification in order to detect the level of the signal amplified by the amp mentioned above. Figure 8 shows this rectifier. By smoothing capacitor after the diode, the pulsating contained in the rectified DC output is removed. In this case, the $C=1000$ $[\mathrm{pF}]$.

(c) Signal detection circuit

To compare a threshold voltage with the level of the signal through the detector circuit mentioned above, we design the comparator shown in Figure 9. In this case, $R_{l}=$ $1[\mathrm{M} \Omega], R_{2}=47[\mathrm{k} \Omega], R_{3}=10[\mathrm{k} \Omega]$, and $C=0.01[\mu \mathrm{F}]$. Set the threshold voltage to 0.2 [V].

We can be obtained from equation (9) is a positive input voltage $\mathrm{V}$

$$
V=\frac{R_{2} \times V_{C C}}{R_{1}+R_{2}}
$$

By inputting the parameters into equation (9), we can get following equation (10).

$$
V=\frac{47 \times 10^{3} \times 5}{47 \times 10^{3}+1 \times 10^{6}}[V]
$$

Thus, the threshold voltage is 0.2 [V] approx.

(d) Ultrasonic propagation time measurement circuit

Figure 10 shows the ultrasonic propagation time measurement circuit. It takes to receive the delivery of ultrasonic sound, using NANDs construct SR flip-flop. That is, the output of the SR flip-flop becomes high until the receiver receives the reflected ultrasonic generated by the transmitter.

Figure 11 shows the timing chart of the ultrasonic propagation time measurement circuit at this time.

The microcomputer counts the time of the $\mathrm{H}$ level of the output shown in Fig. 10. By using the count value, the distance $r[\mathrm{~cm}]$ between the device and the finger can be measured.

\subsection{Dimensional PSD(Position Sensitive Detector)}

In order to measure the 2 dimensional coordinates of the finger, a 2 dimensional position sensitive detector (PSD) shown in Figure 12 is used.

The PSD is a kind of optical sensor using the $\mathrm{Si}$ photodiode. PSD can detect the position of the entering spot light. The feature of PSD is good for position detection in the context of resolution and response.

The PSD generates the electro charge according to the amount of light reaching a light-receiving surface. The PSD has 4 terminals corresponding to the $\mathrm{X}-\mathrm{Y}$ axis. For these terminals, the resistance values from the light-receiving

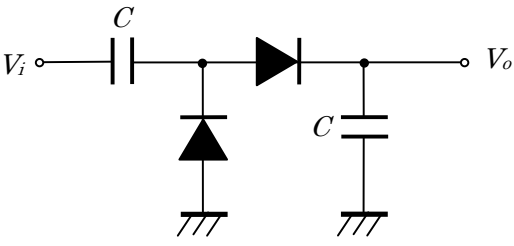

Fig. 8. Detector circuit.

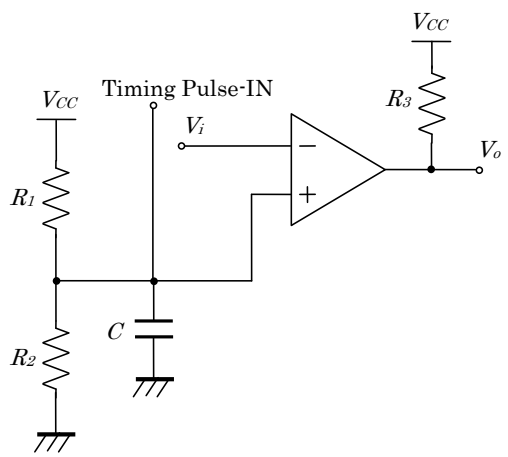

Fig. 9. Signal detection circuit.

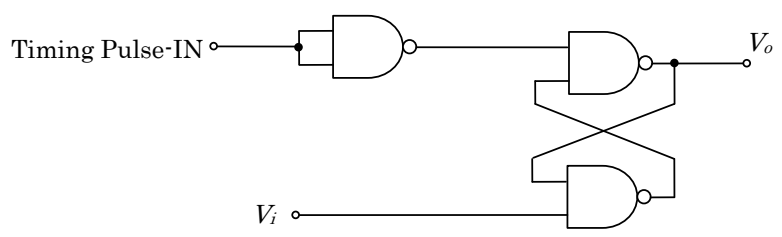

Fig. 10. Ultrasonic propagation time measurement circuit.

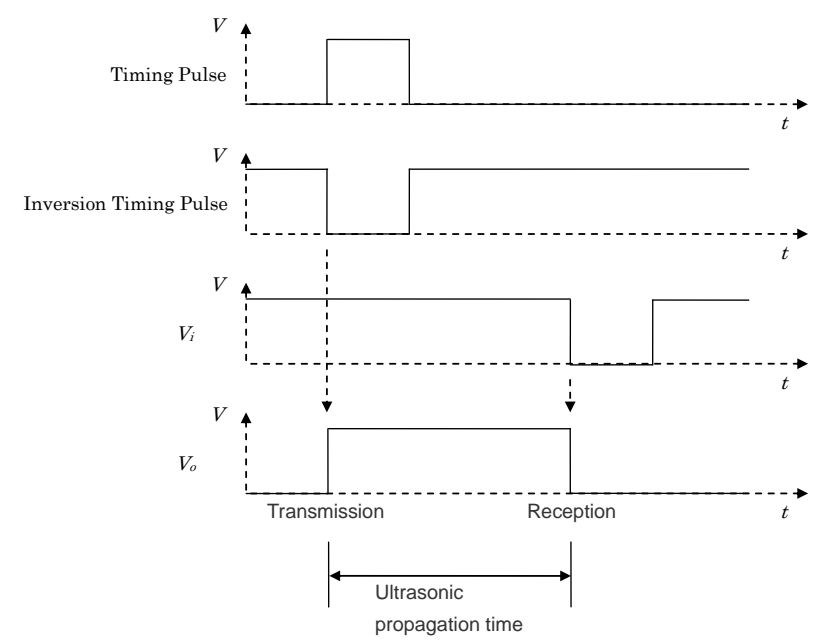

Fig. 11. Time chart of ultrasonic propagation time measurement circuit.

point to each terminal vary according to the lighted position on the PSD. That is, the output currents from 4 terminals are also changing by the lighted position. By using the ration calculated by the currents from 4 terminals, the position of the light spot can be detected as the gravity of the light spot.

In this study, we use the PSD, S2044 of Hamamatsu 
Photonics. Its active area is $4.7 \times 4.7 \mathrm{~mm}$.

In Figure 12, we show coordinates of a center of gravity point as $(x, y)$. According to the center-of-gravity point of this spot light, current value $I_{X 1}, I_{X 2}, I_{Y 1}$, and $I_{Y 2}$ are obtained from the electrode of the four electrodes $X 1, X 2$, $Y 1$, and $Y 2$, respectively. In this case, the $\mathrm{X}$ coordinate of the center of gravity is the following equation.

$$
\frac{\left(I_{X 2}+I_{Y 1}\right)-\left(I_{X 1}+I_{Y 2}\right)}{I_{X 1}+I_{X 2}+I_{Y 1}+I_{Y 2}}=\frac{2 x}{L_{x}}
$$

The following equation will be the $\mathrm{Y}$ coordinate of the center of gravity.

$$
\frac{\left(I_{X 2}+I_{Y 2}\right)-\left(I_{X 1}+I_{Y 1}\right)}{I_{X 1}+I_{X 2}+I_{Y 1}+I_{Y 2}}=\frac{2 y}{L_{y}}
$$

Moreover, since $L_{X}$ and $L_{Y}$ are $L_{X}=5.7 \mathrm{~mm}$ and $L Y=5.7$ $\mathrm{mm}$ in S2044 respectively, the center-of-gravity point coordinates of spot light can be searched for from the conversion type of equations (11) and (12).

The condensed laser beam is reflected by the finger and returns to the PSD through the lens. Figure 13 shows this relationship. Figure 14 is extracted OPAO'P' plane of Figure 13. Since the fixed value D of Figure $14, \theta$ is the angle represented by the following formula.

$$
\theta=\tan ^{-1} \frac{x^{\prime}}{D}
$$

\subsection{Measurement of Two-Dimensional Coordinates of the Finger}

We can calculate the X-Y coordinate by using $\theta$ and $r$ as shown in the following equation.

$$
\begin{aligned}
& x=r \sin \theta \\
& y=r \cos \theta
\end{aligned}
$$

Next, we describe the circuit to extract the modulated

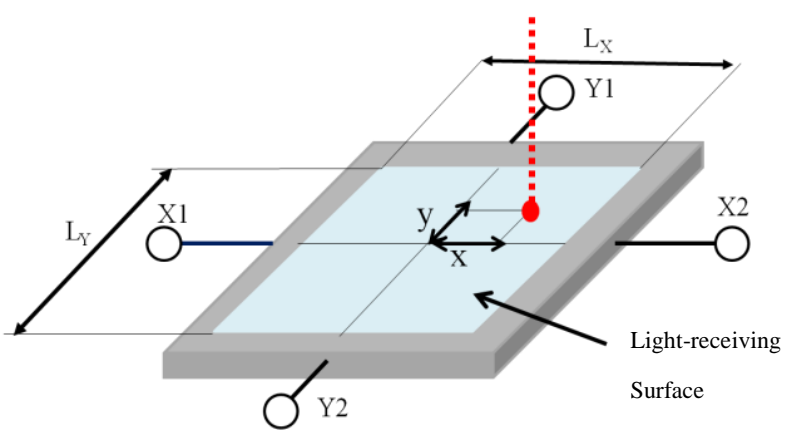

Fig. 12. 2 Dimensional PSD.

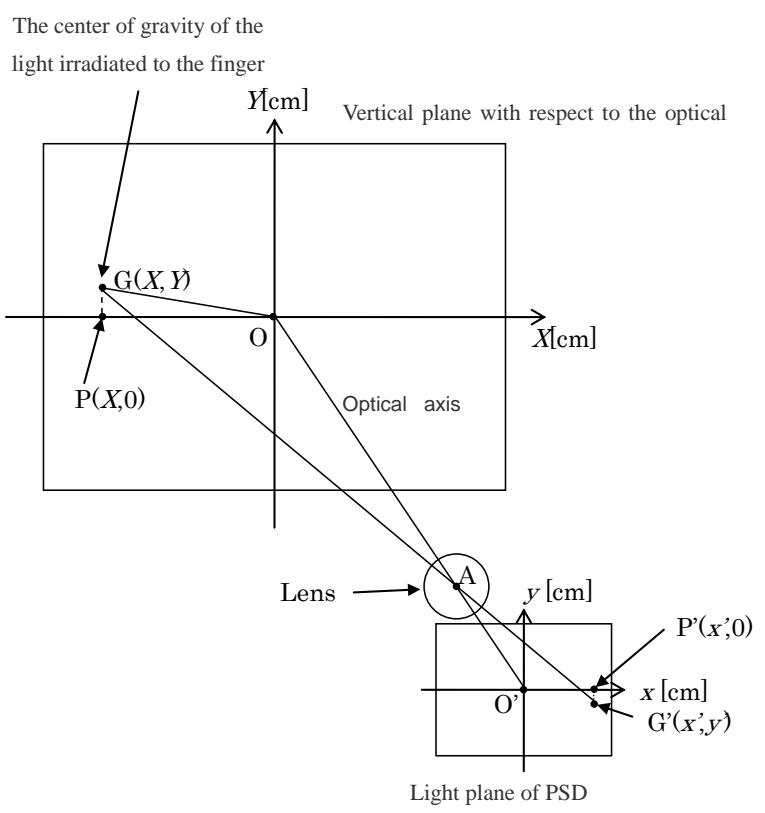

Fig. 13. Relationship diagram of the plane and the light source is present PSD.

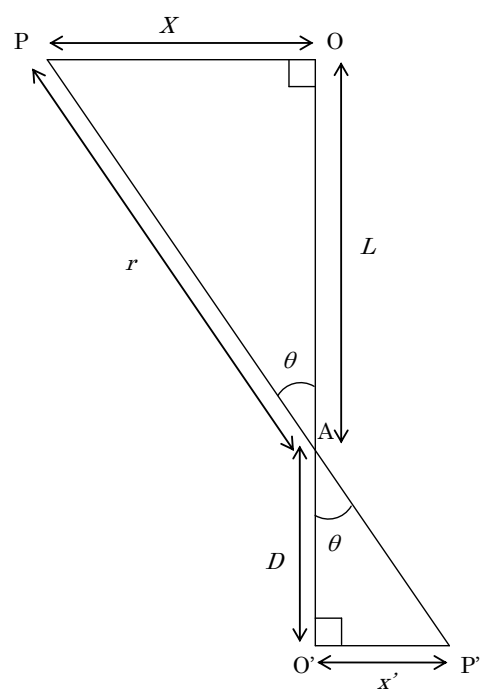

Fig. 14. OPAO'P 'plane

signal from the output signal of the PSD sensor.

(a) Multiple feedback type BPF

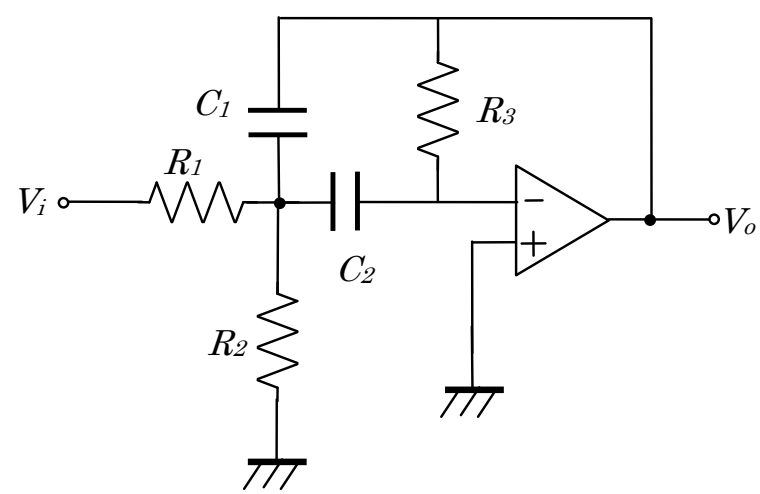

Fig. 15. Multiple feedback type BPF. 
I-V conversion circuit converts the current output from the PSD to the voltage. The center frequency of the laser light using a multiple feedback type BPF shown in Fig 15 is extracted.

After this smoothing, the outputted signal is incorporated in the PC to calculate the coordinates.

\section{Experiments}

\subsection{Experimental Measurement of the Distance $r[\mathrm{~cm}]$ by Ultrasonic Sensors}

We measured the distance $r$ from a finger using an ultrasonic sensor. Figure 16 and Table 1 shows the results. Based upon the foregoing, we can measure the distance to a finger with approximately less than $1 \mathrm{~cm}$ of error.

\subsection{Measurement of the Angle $\theta\left[^{\circ}\right]$ by PSD Sensor}

We measured the angle $\theta$ by PSD sensor. Figure 17 and Table 2 shows the results. Based upon the foregoing, we can measure the argument of the finger at error approximately $3^{\circ}$.

\subsection{Identification Accuracy by Distance $r$ and Angle $\theta$ Coordinates}

Using a distance $r$ measured by the ultrasonic sensor and the angle $\theta$ measured by the PSD sensor, we have identified two-dimensional coordinates. Table 3 shows that accuracy. Based upon the foregoing, we can measure the $x$ and $y$ coordinate of the finger at error about $1.3 \mathrm{~cm}$.

Table 1. The average of the absolute error of $r$ and standard deviation.

\begin{tabular}{|c|c|c|}
\hline & $\begin{array}{c}\text { The average of the } \\
\text { absolute error }[\mathrm{cm}]\end{array}$ & $\begin{array}{c}\text { Standard } \\
\text { deviation }\end{array}$ \\
\hline$r$ & 0.70 & 0.52 \\
\hline
\end{tabular}

Table 2. The average of the absolute error of $\theta$ and standard deviation.

\begin{tabular}{|c|c|c|}
\hline & $\begin{array}{c}\text { The average of the } \\
\text { absolute value of the } \\
\text { error }^{\circ} \text { ] }\end{array}$ & $\begin{array}{c}\text { Standard } \\
\text { deviation }\end{array}$ \\
\hline$\theta$ & 2.99 & 2.42 \\
\hline
\end{tabular}

Table 3. Average of the absolute value of the maximum error and standard deviation of the coordinate $x$ and $y$.

\begin{tabular}{|c|c|c|}
\hline & $\begin{array}{c}\text { The average of the } \\
\text { absolute value of the } \\
\text { maximum error }[\mathrm{cm}]\end{array}$ & $\begin{array}{c}\text { Standard } \\
\text { deviation }\end{array}$ \\
\hline$x$ & 1.34 & 1.30 \\
\hline$y$ & 1.30 & 0.80 \\
\hline
\end{tabular}

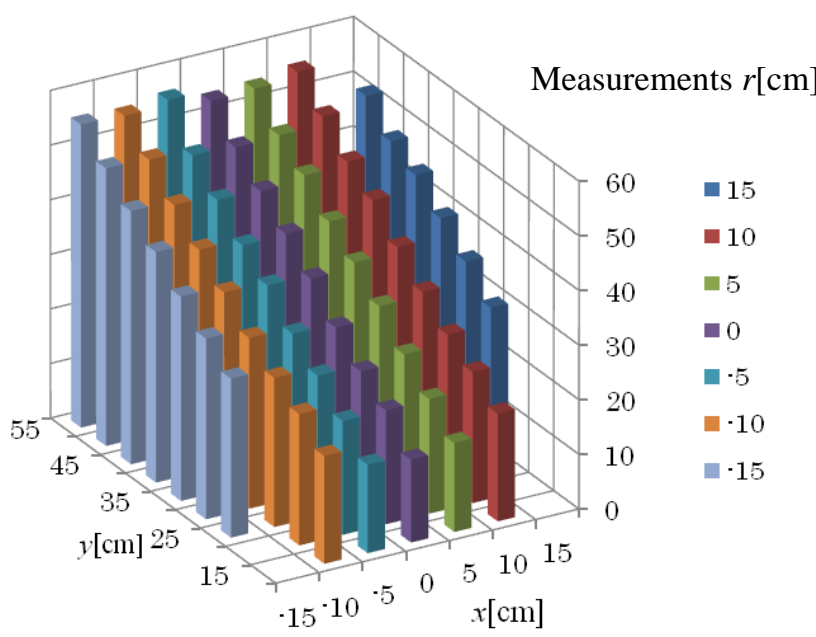

Fig. 16. As a result of the distance $r[\mathrm{~cm}]$ measurement using an ultrasonic sensor.

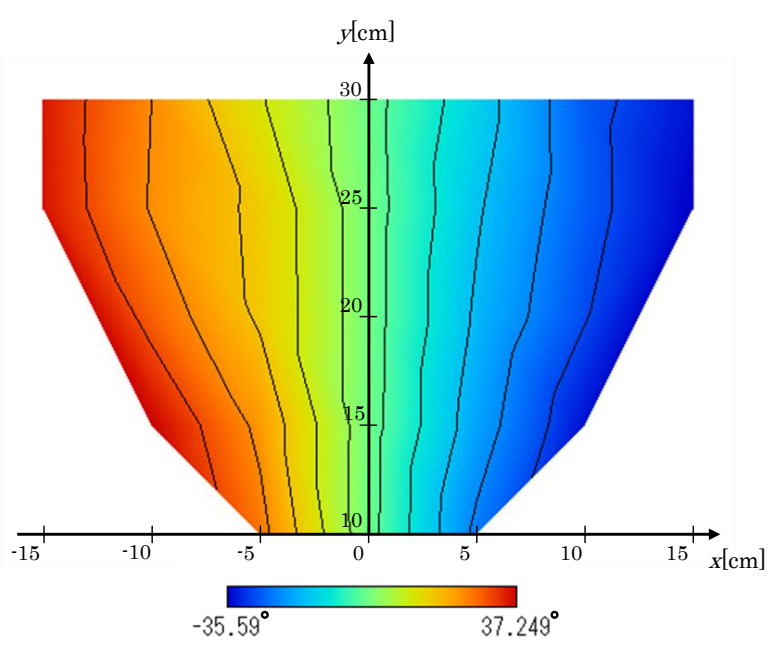

Fig. 17. As a result of the angle $\theta\left[^{\circ} \quad\right]$ measurement using an PSD sensor

\section{Conclusions}

In this study, we were able to determine the two-dimensional coordinates of the finger using the PSD sensor and ultrasonic sensor. So, we make sure that from 
one point of the coordinate detection is possible in principle. In the future, this is applied to the touch panel. Further, as each element is reduced, it is possible to miniaturize the apparatus as well. I think the potential spread of the operation as a device by incorporating a device such as a smart phone it.

\section{References}

[1] Nozomu Matsui, and Yoshikazu Yamamoto : "Virtual Keyboard:Realization of Robust Real-Time Fingertip Detection from Video-Image", Workshop miscellany about the system of programming and the application Japan Society for Software Science and Technology, 2000, Japanese

[2] Carlo Tomasi : "Full-size projection keyboard for handheld devices", Communications of the ACM - A game experience in every application, Vol. 46, pp. 70-75, 2003

[3] Iwao Matuya, Makoto Oshio, Ryota Tomishi, Maya Sato, Kiyoshi Kanekawa, Motoichi Takahashi, Satoru Miura, Yasutsugu Suzuki, Tomohiko Hatada, Ryouta Katamura, Yoshihiro Nitta, Takashi Tanii, Shuichi Shoji, Akira Nishitani, and Iwao Ohdomari : "Noncontact-Type Relative Displacement Monitoring System Using Position Sensitive Detector", Miscellany of Architectural Institute of Japan, Vol. 16, No. 33, pp. 469-472, 2010, Japanese 